\title{
Erratum to: Does Surgically Induced Right Bundle Branch Block Really Effect Ventricular Function in Children After Ventricular Septal Defect Closure?
}

\author{
Cem Karadeniz ${ }^{1} \cdot$ Semra Atalay $^{1} \cdot$ Fikri Demir $^{1} \cdot$ Ercan Tutar $^{1} \cdot$ Omer Ciftci $^{1}$ \\ Tayfun Ucar ${ }^{1} \cdot$ Adnan Uysalel $^{2} \cdot$ Zeynep Eyileten $^{2}$
}

Published online: 21 March 2015

(c) Springer Science+Business Media New York 2015

\section{Erratum to: Pediatr Cardiol (2015) 36:481-488 DOI 10.1007/s00246-014-1037-9}

The abstract and first paragraph of discussion section of the original version of this article unfortunately contained a few mistakes. The corrections are given below:

In the abstract section, the sentence 'When compared to controls, operated group had statistically higher tricuspid annular plane systolic excursion $(p<0.05)$, lower systolic, early diastolic, and late diastolic myocardial velocities, higher left and right ventricular myocardial performance indices, irrespective of the presence of RBBB' should be replaced by 'When compared to controls, operated group had statistically lower tricuspid annular plane systolic excursion $(p<0.05)$, lower systolic, early diastolic, and late diastolic myocardial velocities, higher left and right ventricular myocardial performance indices, irrespective of the presence of RBBB'.

In first paragraph of discussion section, the sentence 'The VSD repair group had significantly higher TAPSE and lower systolic, early diastolic, and late diastolic myocardial velocities, together with higher biventricular MPIs compared with controls, irrespective of the persence of RBBB.' should be replaced by 'The VSD repair group had significantly lower TAPSE and systolic, early diastolic, and late diastolic myocardial velocities, together with higher biventricular MPIs compared with controls, irrespective of the presence of RBBB.'
The online version of the original article can be found under doi:10.1007/s00246-014-1037-9.

Cem Karadeniz

karadenizcem@yahoo.com

1 Department of Pediatric Cardiology, School of Medicine, Ankara University, Cebeci 06620, Ankara, Turkey

2 Department of Cardiovascular Surgery, School of Medicine, Ankara University, Ankara, Turkey 